\title{
Sequence Domain Harmonic Modeling of Type-IV Wind Turbines
}

\author{
Guest, Emerson; Jensen, Kim Høj; Rasmussen, Tonny Wederberg
}

Published in:

IEEE Transactions on Power Electronics

Link to article, DOI:

10.1109/TPEL.2017.2735303

Publication date:

2017

Document Version

Peer reviewed version

Link back to DTU Orbit

Citation (APA):

Guest, E., Jensen, K. H., \& Rasmussen, T. W. (2017). Sequence Domain Harmonic Modeling of Type-IV Wind Turbines. IEEE Transactions on Power Electronics, 33(6), 4934 - 4943.

https://doi.org/10.1109/TPEL.2017.2735303

\section{General rights}

Copyright and moral rights for the publications made accessible in the public portal are retained by the authors and/or other copyright owners and it is a condition of accessing publications that users recognise and abide by the legal requirements associated with these rights.

- Users may download and print one copy of any publication from the public portal for the purpose of private study or research.

- You may not further distribute the material or use it for any profit-making activity or commercial gain

- You may freely distribute the URL identifying the publication in the public portal

If you believe that this document breaches copyright please contact us providing details, and we will remove access to the work immediately and investigate your claim. 


\title{
Sequence Domain Harmonic Modeling of Type-IV Wind Turbines
}

\author{
Emerson Guest, Student Member, IEEE, Kim H. Jensen, and Tonny W. Rasmussen, Member, IEEE
}

\begin{abstract}
A sequence domain (SD) harmonic model of a gridconnected voltage-source converter is developed for decoupling converter generated voltage harmonics from voltage harmonics in the external grid. The modeling procedure includes a derivation of the baseband frequency response for regular-sampled pulsewidth modulation and an analysis of converter generated voltage harmonics due to compensated dead-time. The decoupling capabilities of the proposed the SD harmonic model are verified through a power quality (PQ) assessment of a 3MW Type-IV wind turbine. The assessment shows that the magnitude and phase of low-order odd converter generated voltage harmonics are dependent on the converter operating point and the phase of the fundamental component of converter current respectively. The SD harmonic model can be used to make PQ assessments of Type-IV wind turbines or incorporated into harmonic load flows for computation of $P Q$ in wind power plants.
\end{abstract}

Index Terms-Power quality, Power conversion harmonics, Wind turbine, Pulse-width modulated power converters.

\section{INTRODUCTION}

$\mathbf{T}$ $\mathrm{HE}$ assessment and computation of power quality (PQ) of grid-connected power-electronic devices is an ongoing area of research for the energy industry [1]. The assessment of PQ covers the testing of power electronic devices to quantify their harmonic emissions at different operating points. The computation of PQ concerns calculating harmonic load flow in planned or existing networks of power-electronic devices and passive components. The computation of $\mathrm{PQ}$ in wind power plants (WPPs) is normally undertaken in the planning phase. This is to ensure the proper allocation of passive filters such that the wind farm complies with harmonic emission limits. Obtaining a coherence between the models used to assess wind turbine PQ and the models used to compute system PQ is a common goal for wind turbine manufacturers and operators.

The standard approach for assessing the PQ of commercial wind turbines is based on IEC 61400-21:2008 [2] where a measurement of the harmonic current injected by a wind turbine is used to make a statement about its PQ. Current measurements of DFIG wind turbines have been used to assess the statistics of the magnitude and phase of current harmonics [3],[4], to assess harmonic propagation between wind turbines [5] and to quantify how active power production influences the magnitude of low-order harmonic current injection [6]. Current source models derived from measurements have been used to compute harmonic flows in a DFIG based wind farm

E. Guest and T. W. Rasmussen are with the Center for Electric Power and Energy, Technical University of Denmark, Lyngby DK-2800, Denmark (e-mail: edagu@elektro.dtu.dk; twr@elektro.dtu.dk).

K. H. Jensen is with Siemens Gamesa Renewable Energy, Brande DK-7330, Denmark (e-mail: kim_hoej.jensen@siemens.com).
[7], to compute the aggregated current harmonics in a wind farm connected to a public grid [8] and the importance of correctly modeling collector system when using current source models is identified in [9].

PQ computations based on current source models can be inaccurate due to a misrepresentation of the harmonic sources [1],[5],[10]-[11]. Consequently, a per-phase impedance-based harmonic model of the grid-side voltage-source converter (VSC) of a Type-IV wind turbine was proposed in [12] to calculate the internal voltage source of the converter. Impedancebased models have also been used to study harmonic stability of grid-connected converters [13]-[15], resonance phenomenon [16]-[19], converter impedance shaping [20],[21] and harmonic propagation between converters and the grid [22],[23]. Nonlinearities in $d q$ frame current control have been shown to introduce an unbalanced system within the bandwidth of the current controller, precluding the application of single-input single-output models [15],[24],[25]. One method for treating the unbalanced three-phase system is to transform the control matrices into the $d q$ frame for analysis [26]. Alternatively, harmonic linearization can be employed [27] to transform the control matrices into the sequence domain (SD).

Whilst impedance-based models rely on time averaging to rationalize the PWM process, more advanced frequency domain approaches using harmonic transfer matrices (HTM) [28],[29] model the cross-frequency behavior within VSC control and modulation systems. Alternatively, [30],[31] employ the so-called extended harmonic domain (EHD) to model the inter-modulation effects of PWM in dynamic simulations.

In this paper a SD harmonic model is proposed which augments the SD transfer matrix developed in [27] with a disturbance model to capture converter generated voltage harmonics due to dead-time [32]-[35] and the characteristic switching harmonics [36],[37]. The SD harmonic model connects the assessment and computation of PQ by:

1) establishing a generic framework for decoupling converter generated voltage harmonics from voltage harmonics present in the external grid during the PQ assessment,

2) incorporating magnitude and phase information obtained from the PQ assessment into the computation of system $\mathrm{PQ}$,

3) being tractable for PQ computation consisting of tens to hundreds of wind turbines.

The development of the SD harmonic model includes; an analysis of converter generated voltage harmonics due to compensated dead-time; and a derivation of the baseband frequency response of regular-sampled pulse-width modulation (PWM) to represent the inter-modulation effects in sampled 
PWM systems [38]. The decoupling capabilities of the SD harmonic model will be verified through a PQ assessment of a $3 M W$ Type-IV wind turbine. The results of the assessment will show that the magnitude and phase of low-order odd converter generated voltage harmonics are dependent on converter operating point and the phase of the fundamental component of converter current respectively.

The rest of the paper is organized as follows: the harmonic modeling methods are developed in Section II, the case study on a $3 M W$ Type-IV wind turbine is covered in Section III and the paper is concluded in Section IV.

\section{Harmonic Modeling Methodology}

\section{A. Rationale for the Sequence Domain Harmonic Model}

Table I shows the applicability of various harmonic modeling methods to the assessment and computation of PQ for grid-connected VSCs. The most complete timedomain representation of converters is through full-order electromagnetic transient (EMT) models. EMT models synthesize the exact PWM switching and control functionality at the cost of modeling complexity and long simulation time. Cross-frequency models such as the EHD [30]-[31] or HTMs [28]-[29] can be applied to the computation of VSC PQ but are limited to idealized PWM models. Reduced-order impedance-based models have been applied to the study of harmonic propagation between converters and the grid [22],[23], but are yet to consider converter generated voltage harmonics. Current source models, whilst based on real measurements [3]-[9], have shown poor accuracy when computing PQ in WPPs [1],[5],[10]-[11].

TABLE I: Comparison of harmonic modeling methods applicable to the assessment and computation of PQ. VH-very high, H-high, M-medium, L-low.

\begin{tabular}{|c|c|c|c|c|c|}
\hline Method & 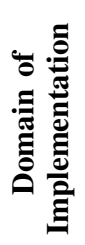 &  &  & 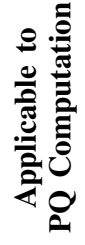 & 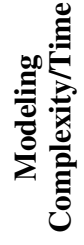 \\
\hline EMT & Time & $x$ & $\checkmark$ & $\checkmark$ & $\mathrm{VH}$ \\
\hline EHD [30]-[31] & Time & $x$ & $x$ & $\checkmark$ & $\mathrm{H}$ \\
\hline HTM [28]-[29] & Freq. & $x$ & $x$ & $\checkmark$ & M \\
\hline Current Inj. [3]-[9] & Freq. & $\checkmark$ & $\checkmark$ & $x$ & $\mathrm{~L}$ \\
\hline Imp. Based [22],[23] & Freq. & $x$ & $x$ & $x$ & $\mathrm{~L}$ \\
\hline Proposed Method & Freq. & $\checkmark$ & $\checkmark$ & $\checkmark$ & $\mathrm{L}$ \\
\hline
\end{tabular}

The proposed SD harmonic model, developed in Section II-B, uses an internal representation of the converter harmonic source to decouple converter generated voltage harmonics from voltage harmonics in the external grid present in the PQ assessment. The ascertained SD harmonic model can then be integrated into harmonic load flows for PQ computations, as required in the planning of WPPs and potentially other renewable energy installations.

\section{B. Modeling Procedure}

A block diagram of a three-phase grid-connected VSC is shown in Fig. 1a. $\mathbf{i}_{a b c}(t)$ and $\mathbf{v}_{a b c}(t)$ are time-domain vectors of the converter line currents and phase-ground voltages at the grid-side of the line inductor $L$ respectively. $\mathbf{v}_{a b c}^{l}(t)$ is the vector of converter load-voltages, $\mathbf{v}_{a b c}^{m}(t)$ is the modulating signal to the PWM block and $\mathbf{s}(t)$ is the vector of gate signals to the VSC. The linearized converter model is shown in Fig. $1 \mathrm{~b}$ for a generic stationary or $d q$ frame current controller with voltage feed-forward such that,

$$
\mathbf{v}_{a b c}^{m}(s)=\mathbf{G}_{a b c} \mathbf{v}_{a b c}(s)+\mathbf{H}_{a b c} \mathbf{i}_{a b c}(s) .
$$

$\mathbf{G}_{a b c}$ and $\mathbf{H}_{a b c}$ are $3 \times 3$ transfer matrices of the voltage feed-forward and current control respectively, including computational and sampling delays. Additionally, the linear part of the modulation process is modeled by a baseband frequency response, $\mathbf{K}_{a b c}$, and the nonlinear part modeled as a disturbance $\mathbf{v}_{a b c}^{d s}(t)$. The disturbance model isolates the converter generated voltage harmonics due to dead-time errorpulses [32]-[35] and the characteristic sideband harmonics [36],[37]. In general the spectrum of $\mathbf{v}_{a b c}^{d s}(t)$ is dependent on the converter operating point, modulation scheme and to a lesser degree, background harmonics in the grid. The block diagram in Fig. $1 b$ is transformed into the SD by the sequence component decomposition, $\mathbf{T}$, defined with respect to the $a$ phase, and the substitution $s=j \omega$,

$$
\begin{aligned}
\mathbf{v}_{p n 0}^{d s}(j \omega)= & \left(\mathbf{I}-\mathbf{T K}_{a b c} \mathbf{G}_{a b c} \mathbf{T}^{-1}\right) \mathbf{v}_{p n 0}(j \omega)+ \\
& \left(-\mathbf{T} \mathbf{K}_{a b c} \mathbf{H}_{a b c} \mathbf{T}^{-1}+j \omega L \mathbf{I}\right) \mathbf{i}_{p n 0}(j \omega) .
\end{aligned}
$$

The transfer matrices $\mathbf{K}_{p n 0} \mathbf{H}_{p n 0}=\mathbf{T K}_{a b c} \mathbf{H}_{a b c} \mathbf{T}^{-1}$ and $\mathbf{K}_{p n 0} \mathbf{G}_{p n 0}=\mathbf{T K}_{a b c} \mathbf{G}_{a b c} \mathbf{T}^{-1}$ are the current controller and voltage feed-forward referred to the sequence domain. $\mathbf{I}$ is the identity matrix. Omitting zero-sequence components and rearranging (2) gives,

$$
\mathbf{v}_{p n}^{d}(j \omega)=\mathbf{Z i}_{p n}(j \omega)+\mathbf{v}_{p n}(j \omega),
$$

with the SD converter impedance,

$$
\mathbf{Z}=\left(\mathbf{I}-\mathbf{K}_{p n} \mathbf{G}_{p n}\right)^{-1}\left(-\mathbf{K}_{p n} \mathbf{H}_{p n}+j \omega L \mathbf{I}\right),
$$

and apparent harmonic voltage source generated by the converter,

$$
\mathbf{v}_{p n}^{d}(j \omega)=\left(\mathbf{I}-\mathbf{K}_{p n} \mathbf{G}_{p n}\right)^{-1} \mathbf{v}_{p n}^{d s}(j \omega) .
$$

Note that $\mathbf{v}_{p n}^{d}(j \omega)$ is acted upon by the voltage feed-forward and thus cannot be directly measured at the converter terminals. $\mathbf{Z}$ has the form,

$$
\mathbf{Z}=\left[\begin{array}{ll}
Z_{p p}(j \omega) & Z_{p n}(j \omega) \\
Z_{n p}(j \omega) & Z_{n n}(j \omega)
\end{array}\right]
$$

Under balanced and slightly unbalanced power system operation $Z_{p n}(j \omega)=Z_{n p}(j \omega) \approx 0$ [27]. In general, $Z_{p p}(j \omega) \neq$ $Z_{n n}(j \omega)$ for $d q$ frame current controllers within the bandwidth of the current controller [24]-[27] and for stationary frame current control when impedance shaping techniques for damping of power system current or voltage harmonics are 


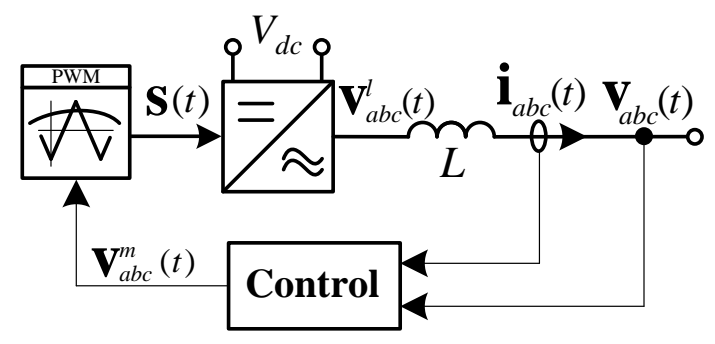

(a) Three-phase grid-connected VSC.

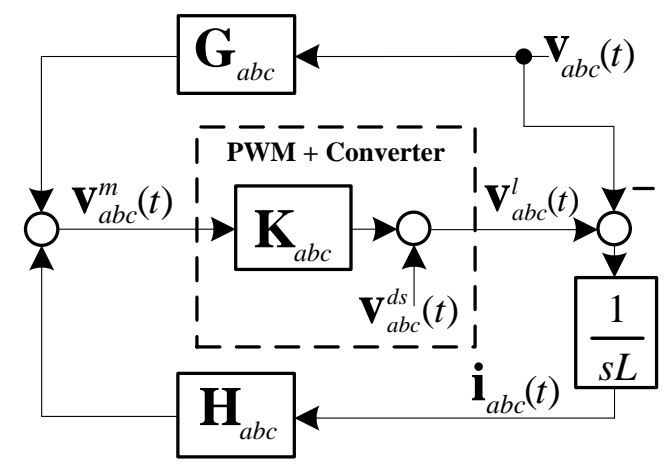

(b) Linearized VSC model containing a PWM model, disturbance model and control transfer functions.

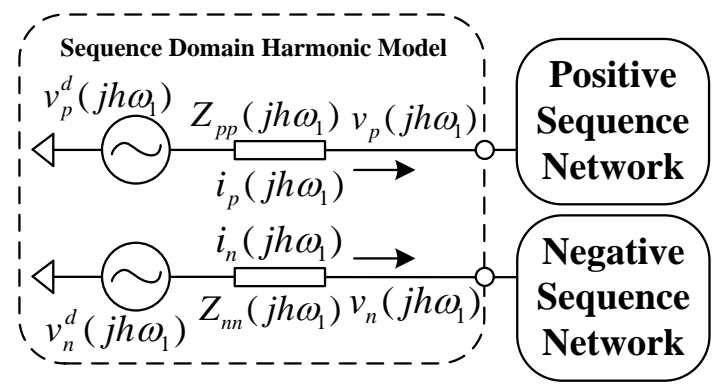

(c) SD harmonic model of VSC connected to an arbitrary linear network for PQ computation.

Fig. 1: Development of the SD harmonic model for the assessment and computation of PQ.

used [21]. The SD harmonic model is obtained by evaluating (3) at harmonic frequencies $\omega=h \omega_{1}$ giving,

$\mathbf{v}_{p n}^{d}\left(j h \omega_{1}\right)=\left[\begin{array}{cc}Z_{p p}\left(j h \omega_{1}\right) & 0 \\ 0 & Z_{n n}\left(j h \omega_{1}\right)\end{array}\right] \mathbf{i}_{p n}\left(j h \omega_{1}\right)+\mathbf{v}_{p n}\left(j h \omega_{1}\right)$,

where $\omega_{1}$ is the fundamental frequency of the power system and $h=2, \ldots, h_{\max }$ are the harmonic orders of interest. Equation (7) is depicted in Fig. 1c as two decoupled Thevenin equivalent circuits, one set for the positive sequence and negative sequence harmonic components respectively. This is a generalization of the harmonic model proposed in [12] which is based on a per-phase equivalent circuit. The need for a SD harmonic model becomes clear when $Z_{p p}\left(j h \omega_{1}\right) \neq$ $Z_{n n}\left(j h \omega_{1}\right)$ or $\mathbf{v}_{p n}^{d}(j \omega)$ contains unbalanced harmonic phasors. Both situations can occur in a typical grid-connected converter application.

\section{Baseband Frequency Response of a Regular-Sampled Pulse-Width Modulator}

Harmonics enter the converter control paths due to the existence of both converter generated and grid voltage harmonics. Therefore, the converter modulating signals, $\mathbf{v}_{a b c}^{m}(t)$, are in general multi-tone signals consisting of a fundamental and harmonic components. Regular-sampled PWM subject to a multi-tone modulating signal cannot be treated as a linear amplifier due to inter-modulation in the baseband range [38]. The continuously-sampled zero-order hold ( $\mathrm{ZOH})$ with frequency response,

$$
K_{Z O H}(j \omega)=\operatorname{sinc}\left(\omega T_{s} / 2\right) e^{-j \omega T_{s} / 2},
$$

is sometimes used to model the low-frequency behavior of the PWM process [14],[27] but does not consider intermodulation effects. Consequently, a baseband frequency response of regular-sampled PWM, $K_{M}(j \omega)$, is derived in the Appendix which for asymmetrical regular-sampled PWM gives,

$$
K_{M}(j \omega)=J_{0}\left(\omega M_{1} T_{s} / 2\right) e^{-j \omega T_{s} 2} .
$$

$M_{1}$ is the magnitude of the fundamental component in the modulating signal, $T_{s}$ is the sampling period and $J_{0}(z)$ is the 0-th order Bessel function of the 1st kind. Note that (9) is a function of $M_{1}$ which accounts for the inter-modulation between the fundamental and harmonic components in the modulating signal. As $M_{1} \approx 1$ for grid-connected VSCs the difference between (8) and (9) is the roll-off of $\operatorname{sinc}(z)$ compared to $J_{0}(z)$.

Equation (9) was verified by a PSCAD (EMTDC) switching model of a two-level VSC. The model used a constant $1100 \mathrm{~V}$ DC link voltage, a pulse ratio $p=\omega_{c} / \omega_{1}=50$, a balanced three-phase passive load and a modulation index ${ }^{1}$ set at $M_{1}=1$. The small-signal gain of the normalized output load voltage to the input modulating signal was measured. A $5 \mu \mathrm{s}$ simulation time-step was found to be sufficient for model accuracy. Fig. 2 shows how $\left|K_{M}(j \omega)\right|$ and $\left|K_{Z O H}(j \omega)\right|$ diverge with frequency. This suggests the importance of using the actual frequency response of the modulator given in (9), when the calculation of higher frequency harmonic components generated by the converter are of interest. Furthermore, the exact baseband response of the converter may improve impedance shaping techniques that rely on algebraic approximation of converter impedance, particularly for shaping in the midband range [21].

Harmonics in the modulating signal due to converter and grid voltage harmonics also produce cross-frequency and cross-sequence harmonic sideband responses in the converter load voltages. The cross-frequency and cross-sequence properties of the harmonic sideband responses preclude linear system modeling (but could be captured with a HTM similar to those used in [28]-[31]). Fortunately, the harmonic sideband

\footnotetext{
${ }^{1}$ A triplen third-harmonic component of magnitude $M_{1} / 6$ was also added to the modulating signal to extend the modulator's linear range to 1.15 . Strictly speaking a factor of $J_{0}\left(\omega \frac{M_{1} T_{c}}{24}\right)$ due to the third harmonic component should be added to (9), however, it has negligible impact on $K_{M}(j \omega)$ over the frequency range of interest.
} 


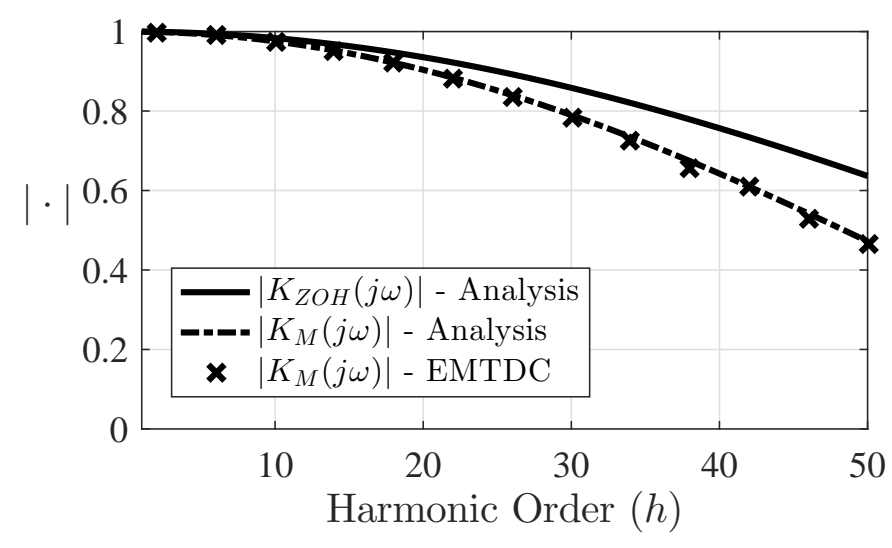

Fig. 2: Modulator gains for asymmetrically regular-sampled PWM and continuously-sampled ZOH when $p=\omega_{c} / \omega_{1}=50$.

responses are considerably smaller in magnitude than the baseband response and are thereby ignored for a significant model simplification $^{2}$. When the SD harmonic model is applied to a VSC the harmonic sideband responses are captured in the apparent harmonic voltage source, $\mathbf{v}_{p n}^{d}(j \omega)$.

\section{Deriving the Converter Impedance}

The converter impedance, $\mathbf{Z}$, required for the case study on a Type-IV wind turbine in Section III is now used to exemplify the deviation that can occur between SD converter impedances. $\mathbf{Z}$ is calculated using (4) and knowledge of the VSC specific control and modulation transfer matrices $\mathbf{G}_{p n}$, $\mathbf{H}_{p n}$ (not provided herein) and $\mathbf{K}_{p n}$ (see (19) in the appendix) respectively. The analytical converter impedance is verified against an empirical result extracted by applying a harmonic perturbation [40] to an EMTDC model $^{3}$ of the wind turbine electrical drive-train, control software implementation and grid connection. The deviation between $\left|Z_{p p}(j \omega)\right|$ and $\left|Z_{n n}(j \omega)\right|$ in the low-harmonic range, shown in Fig. 3, aligns with similar analyses found in [18],[27]. Resonances occurring in the lowharmonic range are found in WPPs [18],[19] and systems connected through long AC cables [16],[17]. The difference between $\left|Z_{p p}(j \omega)\right|$ and $\left|Z_{n n}(j \omega)\right|$ and a system resonance in the low-harmonic range can cause sequence dependent harmonic current amplification [27].

\section{E. Magnitude and Phase of Converter Generated Odd Voltage Harmonics due to Dead-time Error Pulses}

The apparent harmonic voltage source, $\mathbf{v}_{p n}^{d}\left(j h \omega_{1}\right)$, captures the converter generated voltage harmonics. The characteristic sideband harmonics that appear around multiples of the switching frequency, $f_{c}$, are primarily defined by the modulation scheme and the fundamental component in the modulation signal [36],[37]. Within the linear modulating range, low-order

\footnotetext{
${ }^{2}$ Closed-form expressions for the harmonic sideband responses can be derived from (14) and (15) in the Appendix.

${ }^{3}$ In reality the high-power converter introduces low-order voltage harmonics that can distort the converter impedance measurement. The simulation environment has an advantage because nonlinear PWM effects can be minimized.
}

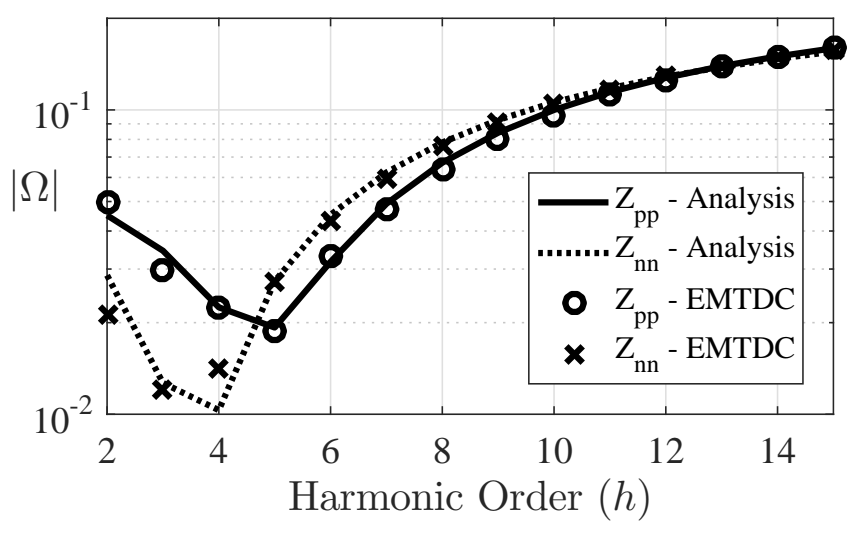

Fig. 3: Magnitude of converter impedances, $\left|Z_{p p}(j \omega)\right|$ and $\left|Z_{n n}(j \omega)\right|$, calculated analytically and extracted empirically from a simulation model.

harmonics ( $\left.f \leq f_{c} / 2\right)$ are predominately contributed by deadtime [32],[35]. In high-power applications the dead-time delay, $T_{d}$, is typically in the range of $1 \%-3 \%$ of the switching period, $T_{c}$.

In uncompensated dead-time (UDT) the rising edge on each switching device is delayed by $T_{d}$ and during the dead-time interval the converter terminal voltage clamps to either the $+V_{d c}$ or $-V_{d c}$ rail depending on the polarity of the load current. If the clamped voltage differs from the ideal voltage then an error pulse of height $V_{d c}$ and width $T_{d}$ occurs. The error pulses due to UDT and sinusoidal load currents have been shown using averaging [32]-[34] to approximate a square wave with complex Fourier coefficients,

$$
U_{h}=\frac{2 V_{d c} T_{d}}{j h \pi T_{c}} .
$$

The harmonic generating effects of compensated dead-time (CDT), however, are relatively undocumented. Therefore, a closer look will be given to CDT as it is often incorporated into high-power converters such as that found in the $3 \mathrm{MW}$ Type-IV wind turbine studied in Section III. In CDT the active switching edge is either delayed or advanced by $T_{d}$ depending on the polarity of the sampled converter current [33]. With sinusoidal load currents the resultant switching edges are theoretically ideally placed. In practice the converter load currents are not sinusoidal and can exhibit multiple zerocrossings due to a superposition of the switching ripple and the fundamental component. The multiple zero-crossings of the load current can cause the dead-time to be misplaced resulting in error pulses. This is shown in Fig. 4 for the $a$-phase rising current, $i_{a}(t)$, where the polarity of the current, $\operatorname{sgn}\left(i_{a}(t)\right)$, changes sign after sampling but before the switching edge. The dead-time is placed on the wrong switching device resulting in an incorrect phase-neutral terminal voltage $v_{a n}^{l}(t)$ and a nonzero error pulse signal $e_{a}(t)$. The resultant train of error pulses tend to have the same polarity as the fundamental load current, $\operatorname{sgn}\left(i_{a, 1}(t)\right)$. This is opposite to what occurs in UDT where the error pulses have the opposite polarity to the fundamental load current [32]-[34]. Moreover, the error pulses due to CDT are centered around the fundamental current zero-crossing. 


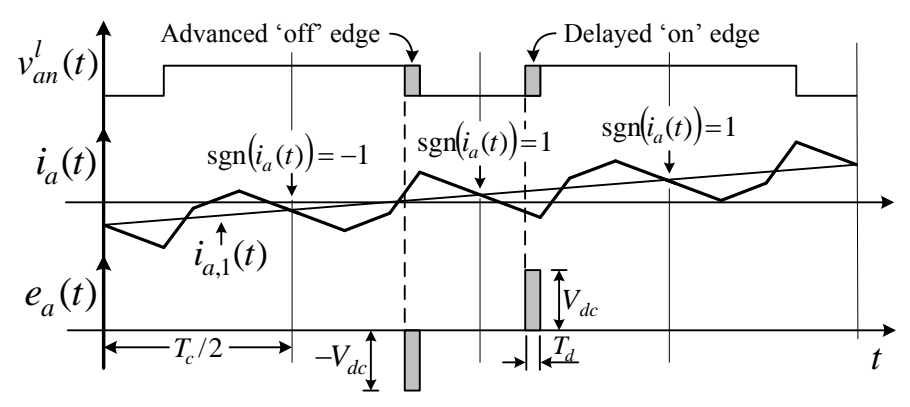

Fig. 4: Generation of the $a$-phase to neutral error pulse signal, $e_{a}(t)$, due to the load current, $i_{a}(t)$, changing polarity after sampling but before the switching edge.

A challenge with modeling CDT is that the placement of the error pulses is a function of both the instantaneous switching time of the PWM scheme and the polarity of the load current response. EMTDC simulations can handle such nonlinearity because the system matrices are updated at each time-step. Cross-frequency models such as HTMs [28]-[29] or the EHD [30]-[31] cannot, at this time, handle such nonlinearity. A simplified model is obtained by assuming an equal number of error pulses $k_{d} \in \mathbb{N}$ occur either side of the fundamental current zero-crossing. Averaging over each switching period, $T_{c}$, gives the averaged error pulse signal, $\bar{e}_{a}(t)$, with averaged error pulses of height $V_{d c} D$, where $D=T_{d} / T_{c}$, and width $k_{d} T_{c}$ as shown in Fig. 5. The phase of $\bar{e}_{a}(t)$ is determined by the phase of the fundamental component of current, $\theta_{i, 1}$. The averaged error pulse signals for the $b$ - and $c$-phases are obtained by a $-120^{\circ}$ and $-240^{\circ}$ phase shift respectively.

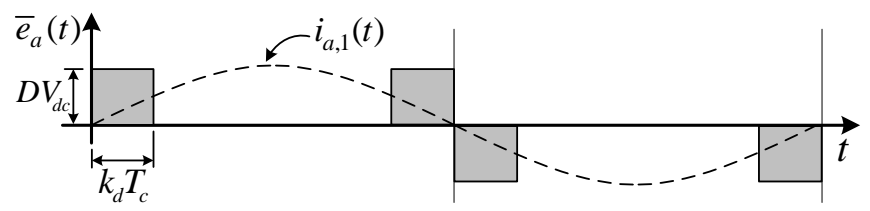

Fig. 5: Averaged $a$-phase to neutral error pulse signal, $\bar{e}_{a}(t)$, for $k_{d}$ error pulses either side of the $a$-phase fundamental current zero-crossing.

For balanced load currents, $\overline{\mathbf{e}}_{a b c}(t)$ will be balanced and produce characteristic power system harmonics [1] where all non-zero positive and negative sequence odd harmonics have order $h=6 k+1$ and $h=6 k-1$ respectively, where $k \in \mathbb{N}$. From Fig. 5 the complex Fourier coefficients, $C_{h}$, of $\bar{e}_{a}(t)$ are,

$$
\begin{aligned}
C_{h} & =\frac{\omega_{1}}{2 \pi} \int_{\theta_{i, 1} / \omega_{1}}^{T_{1}+\theta_{i, 1} / \omega_{1}} \bar{e}_{a}(t) e^{-j h \omega_{1} t} d t \\
& =\frac{2 V_{d c} D}{h \pi}\left[1-\cos \left(k_{d} \frac{2 \pi h}{p}\right)\right] e^{j h \theta_{i, 1},}
\end{aligned}
$$

where $p=\omega_{c} / \omega_{1}$ is the pulse ratio and $h$ is odd. The active/reactive power injection (converter operating point) in a wind turbine will determine the magnitude of the fundamental load currents and influence the number of current zero-crossings. Consequently, (11), being a function of $k_{d}$, indicates that the low-order voltage harmonics generated by the converter due to error pulses will vary with the converter operating point. Furthermore, the phase of the error pulse harmonics are seen to be linearly related to the phase of the fundamental component of current as $h \theta_{i, 1}$.

Fig. 6 shows the magnitude spectrum ${ }^{4}, 2\left|C_{h}\right|$, for different values of $k_{d}$ when $D=0.01, V_{d c}=1100$ and $p=50$. The largest spectral components appear at harmonic multiples of the fundamental. This aligns with the conventional notion of using CDT to improve converter performance at the fundamental frequency [32],[33]. It is apparent that the harmonic order with the largest magnitude moves closer to the fundamental as $k_{d}$ increases. This indicates that lower-order harmonics will tend to increase with decreasing active power injection. Also shown in Fig. 6 is the envelope of $C_{h}$ calculated as,

$$
\max _{k_{d}}\left(\left|C_{h}\right|\right)=\frac{4 V_{d c} D}{h \pi}=2 \cdot\left|U_{h}\right| .
$$

Equation (12) indicates that across a range of converter operating points (values of $k_{d}$ ) the envelope of $C_{h}$ is twice that of $U_{h}$ given in (10). Therefore, CDT can introduce harmonics of significantly larger magnitude than UDT.



Fig. 6: Magnitude of voltage harmonics due to averaged error pulses, $2\left|C_{h}\right|$, for different operating points (values of $k_{d}$ ).

In general there will not be identically $k_{d}$ error pulses either side of the fundamental zero crossing. In some cases an error pulse will be dropped or an error pulse will be added, resulting in an unbalanced three-phase signal and thereby introducing even and non-characteristic odd harmonics. The precise arrangement and quantity of the error pulses is a function of the phase of the PWM carrier wave, $\phi_{c}$.

\section{Case Study - Sequence Domain Harmonic MODEL OF A 3MW TYPE-IV WIND TURBINE}

Two SD harmonic model instances were obtained for a $3 M W$ Type-IV wind turbine converter with the ratings given in Table II. The first instance was derived from voltage and current measurements of the actual wind turbine connected to the Danish power grid. The instance was derived from simulated data taken from an EMTDC model of the wind turbine, connected to a lumped grid model (short-circuit

\footnotetext{
${ }^{4}$ The factor 2 is required to obtain the magnitude of the real Fourier coefficients.
} 
ratio of 10) containing low-order voltage harmonics accruing $1.5 \%$ total harmonic distortion and representing a mildly distorted grid [39]. The EMTDC model consisted of reducedorder mechanical model and a switching model for the converter including the control software and the dead-time compensation scheme. The grid connection in the EMTDC model was intentionally chosen to differ from the measured grid. This is to emphasize the decoupling capabilities of the SD harmonic model under different grid conditions.

TABLE II: Ratings of the Type-IV wind turbine grid-side VSC.

\begin{tabular}{|l|l|l|}
\hline Parameter & Value & Symbol \\
\hline Rated Active Power & $3 \mathrm{MW}$ & $P_{\text {rated }}$ \\
\hline Rated Frequency & $50 \mathrm{~Hz}$ & $f_{1}$ \\
\hline Rated Voltage (line-line) & $690 \mathrm{~V}_{R M S}$ & - \\
\hline Switching Frequency & $2.5 \mathrm{kHz}$ & $f_{c}$ \\
\hline Sampling Frequency & $5 \mathrm{kHz}$ & $f_{s}$ \\
\hline Dead-time Delay & $4 \mu \mathrm{s}$ & $T_{d}$ \\
\hline Dead-time Scheme & $\mathrm{CDT}$ & - \\
\hline
\end{tabular}

\section{A. Harmonic Measurement Procedure}

The harmonic measurement procedure applied to the wind turbine was based on [2] and is summarized as follows:

1) Set the first operating point as $P=0.1 \cdot P_{\text {rated }}, Q \approx 0$, where $P, Q$ are the active and reactive power injections respectively.

2) Sample $\mathbf{v}_{a b c}(t), \mathbf{i}_{a b c}(t)$ (see Fig. 1a) at $20 k H z$ for $600 s$.

3) Repeat step 2) for the remaining operating points i.e. $P=$ $0.2 \cdot P_{\text {rated }}, \ldots, 1 \cdot P_{\text {rated }}, Q \approx 0$.

4) Use the fundamental zero-crossings of $i_{a}(t)$ to partition samples into blocks of data corresponding to 10 fundamental cycles (12 cycles for $60 \mathrm{~Hz}$ systems).

5) Calculate the FFT for each block of samples to obtain the SD phasors $\mathbf{v}_{p n}\left(j h \omega_{1}\right)$ and $\mathbf{i}_{p n}\left(j h \omega_{1}\right)$.

6) Calculate $\mathbf{v}_{p n}^{d}\left(j h \omega_{1}\right)$ with (7).

\section{B. Decoupling of the Apparent Harmonic Voltage Source for $P=0.5 \cdot P_{\text {rated }}$}

The 5th and 7 th harmonic current phasors, $i_{n}\left(j 5 \omega_{1}\right)$ and $i_{p}\left(j 7 \omega_{1}\right)$, and apparent harmonic voltage phasors, $v_{n}^{d}\left(j 5 \omega_{1}\right)$ and $v_{p}^{d}\left(j 7 \omega_{1}\right)$, for each block of data outputted by the FFT were chosen to demonstrate the decoupling capabilities of the SD harmonic model when $P=0.5 \cdot P_{\text {rated }}$. Fig. 7 shows that the EMTDC and measured data for $i_{n}\left(j 5 \omega_{1}\right)$ and $i_{p}\left(j 7 \omega_{1}\right)$ differ in both amplitude and phase, which is expected because the current response depends on the converter voltage, the grid voltage and the interconnecting impedance. The EMTDC and measured data for $v_{n}^{d}\left(j 5 \omega_{1}\right)$ and $v_{p}^{d}\left(j 7 \omega_{1}\right)$, however, are approximately equal in both magnitude and phase. Moreover, their phase is very close to $0^{\circ}$. This is expected because the fundamental current zero-crossing was used to synchronize the sampling, i.e. $\theta_{i, 1} \approx 0^{\circ}$, and the phase of the low-order harmonics due to dead-time error pulses was shown in Section II-E to be $h \theta_{i, 1}$, therefore, $h \theta_{i, 1} \approx 0^{\circ}$.

In contrast to the phase-locked harmonics shown in Fig. 7, the fixed frequency carrier sideband harmonic $v_{p}^{d}\left(j 48 \omega_{1}\right)$,
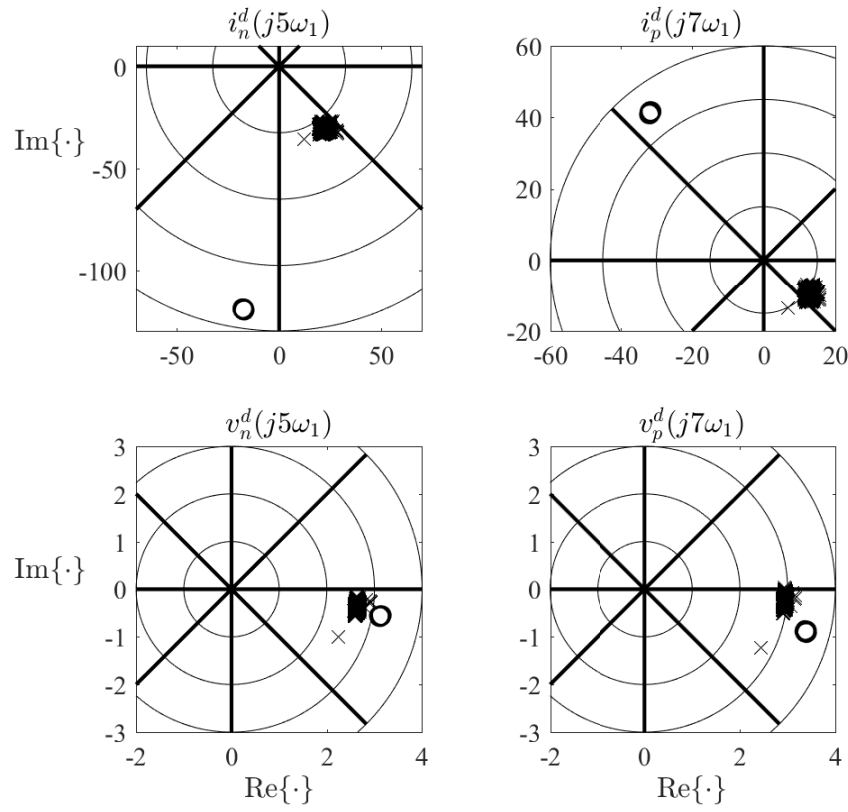

Fig. 7: Phasor diagrams for the converter currents $i_{n}\left(j 5 \omega_{1}\right)$, $i_{p}\left(j 7 \omega_{1}\right)$ and apparent harmonic voltage sources $v_{n}^{d}\left(j 5 \omega_{1}\right)$, $v_{p}^{d}\left(j 7 \omega_{1}\right)$ when $P=0.5 \cdot P_{\text {rated }}$. EMTDC data (०), measured data $(\times)$.

shown in Fig. 8, exhibits uniformly distributed phase over $\left[\begin{array}{ll}-180^{\circ} & 180^{\circ}\end{array}\right]$. The phase distribution is because the grid frequency, estimated as $f_{1}=49.9625 \mathrm{~Hz}$, causes a frequency error between the 48th bin of the FFT, located at $48 f_{1}=$ $2398.2 \mathrm{~Hz}$, and the fixed frequency of the sideband harmonic, located at $f_{c}-2 f_{1}=2400.075 \mathrm{~Hz}$. It was mentioned in Section II-E that non-symmetry in the dead-time error pulses would introduce even and non-characteristic odd harmonics that are a function of PWM carrier phase $\phi_{c}$. An example of this is shown in Fig. 8 for $v_{p}^{d}\left(j 6 \omega_{1}\right)$ which also exhibits uniformly distributed phase over $\left[-180^{\circ} 180^{\circ}\right.$. In fact, all even and non-characteristic odd harmonics at all operating points tend to exhibit this phase distribution. A similar observation is noted in [11].
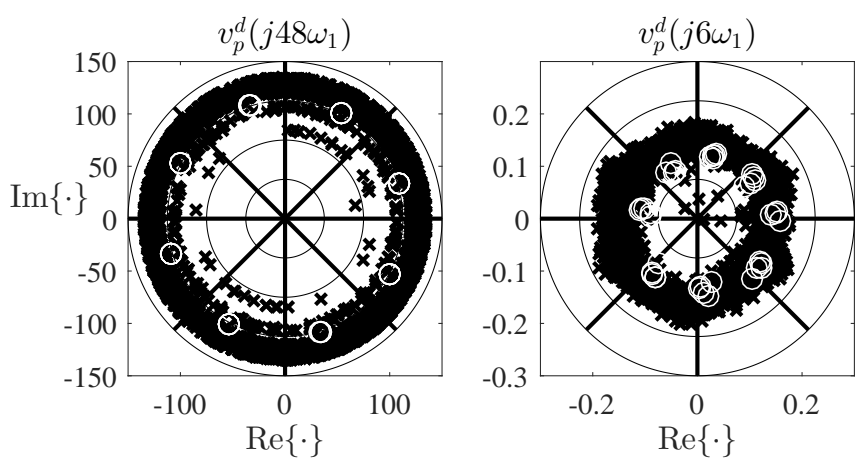

Fig. 8: Phasor diagrams for the apparent harmonic voltage sources $v_{p}^{d}\left(j 48 \omega_{1}\right)$ and $v_{p}^{d}\left(j 6 \omega_{1}\right)$ when $P=0.5 \cdot P_{\text {rated }}$. EMTDC data (o), measured data $(\times)$. 
C. Magnitude and Phase of the Apparent Harmonic Voltage Source vs Active Power

Section II-E identified that characteristic harmonics due to dead-time error pulses will express themselves in $\mathbf{v}_{p n}^{d}\left(j h \omega_{1}\right)$ as low-order positive and negative sequence harmonics of order $h=6 k+1$ and $h=6 k-1$ respectively, where $k \in \mathbb{N}$. Fig. 9 shows how the magnitudes, $\left|v_{n}^{d}\left(j(6 k-1) \omega_{1}\right)\right|$ and $\left|v_{p}^{d}\left(j(6 k+1) \omega_{1}\right)\right|^{5}$ for $1 \leq k \leq 4$, vary with active power injection. The alignment between the EMTDC and measured data reinforces how a common SD harmonic model of the converter can be extracted regardless of the external grid conditions. This is not possible using only current measurement as shown in Section III-B. Fig. 6 indicated that $\left|v_{n}^{d}\left(j 5 \omega_{1}\right)\right|$ and $\left|v_{p}^{d}\left(j 7 \omega_{1}\right)\right|$ should increase for increasing $k_{d}$ (decreasing active power) which aligns with Fig. 9. Similarly, Fig. 6 indicated that $\left|v_{n}^{d}\left(j 11 \omega_{1}\right)\right|$ and $\left|v_{p}^{d}\left(j 13 \omega_{1}\right)\right|$ should have their largest magnitude at a lower value of $k_{d}$, also reflected in Fig. 9. Similar conclusions can be drawn for $\left|v_{n}^{d}\left(j 17 \omega_{1}\right)\right|$ to $\left|v_{p}^{d}\left(j 25 \omega_{1}\right)\right|$.
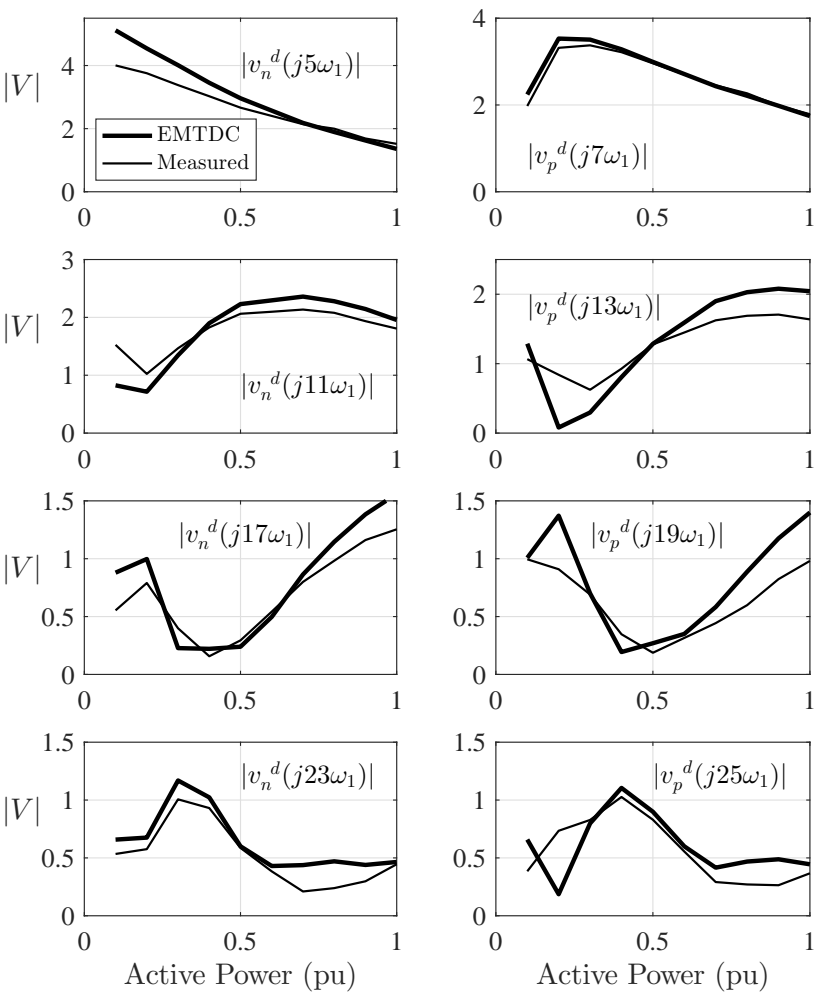

Fig. 9: Magnitude of the apparent harmonic voltage source, $\left|v_{n}^{d}\left(j(6 k-1) \omega_{1}\right)\right|$ and $\left|v_{p}^{d}\left(j(6 k+1) \omega_{1}\right)\right|$ for $1 \leq k \leq 4$, as a function of active power injection.

Fig. 10 shows how the phases, $\arg \left(v_{n}^{d}\left(j(6 k-1) \omega_{1}\right)\right)$ and $\arg \left(v_{p}^{d}\left(j(6 k+1) \omega_{1}\right)\right)$ for $1 \leq k \leq 4$, vary with active power injection. It was commented in Section III-B that as $\theta_{i, 1} \approx 0^{\circ}$ the phases of the low-order harmonics due to dead-time should be $h \theta_{i, 1} \approx 0^{\circ}$. Fig. 10 shows that $\arg \left(v_{n}^{d}\left(j 5 \omega_{1}\right)\right)$ to

${ }^{5}$ To improve clarity only the mean value of amplitude is plotted. $\arg \left(v_{p}^{d}\left(j 13 \omega_{1}\right)\right)$ remain close to $0^{\circ}$ across all operating points (with some deviation occurring for lower active power bins). The phases of the higher harmonic orders, $\arg \left(v_{n}^{d}\left(j 17 \omega_{1}\right)\right)$ to $\arg \left(v_{p}^{d}\left(j 25 \omega_{1}\right)\right)$, remain approximately in the first quadrant $\left[-45^{\circ} 45^{\circ}\right]$. The deviations between the phases obtained from the EMTDC and measured data can result from unmodeled PWM behavior, differences in the PWM carrier phase, and differences in the instantaneous sampling of the voltages and currents used to calculate $\mathbf{v}_{p n}^{d}\left(j h \omega_{1}\right)$ in the two modeling instances.
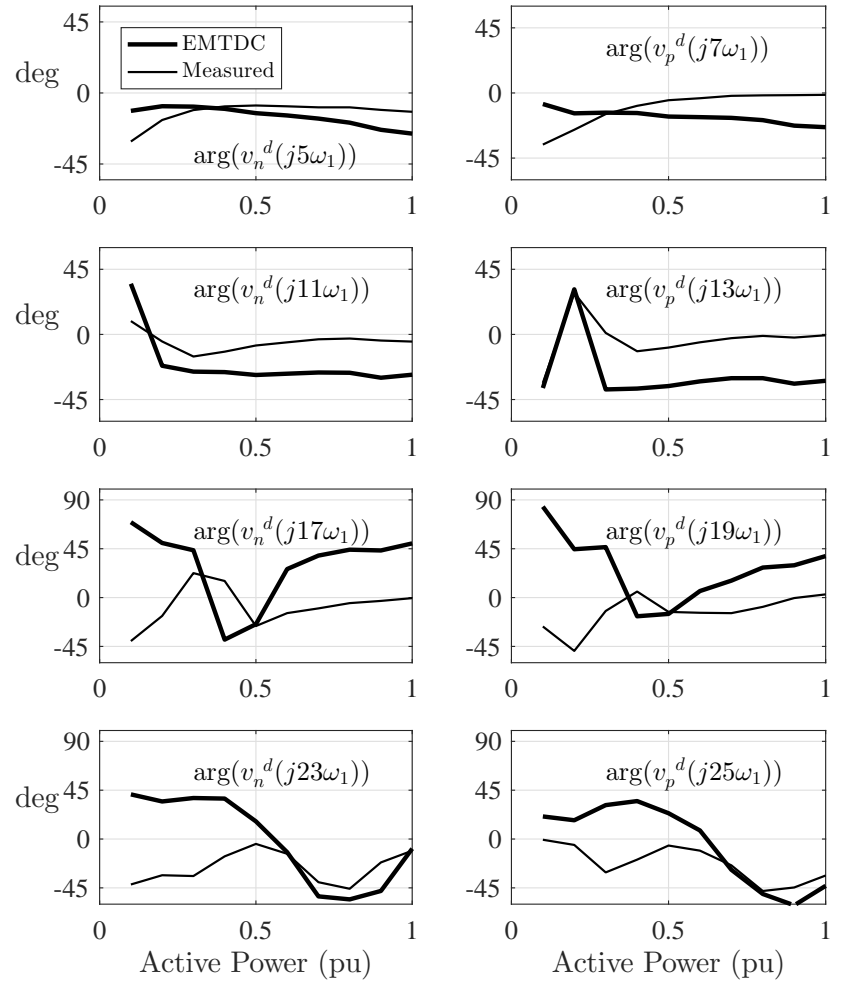

Fig. 10: Phase of the apparent harmonic voltage source, $\arg \left(v_{n}^{d}\left(j(6 k-1) \omega_{1}\right)\right)$ and $\arg \left(v_{p}^{d}\left(j(6 k+1) \omega_{1}\right)\right)$ for $1 \leq k \leq 4$, as a function of active power injection.

Fig. 11 shows a virtual spectrum of the worst-case loworder harmonics obtained by taking the maximal elements of $\left|\mathbf{v}_{p n}^{d}\left(j h \omega_{1}\right)\right|$ from each operating point. Overlaid on Fig. 11 is the envelope of the averaged CDT model, $\max _{k_{d}}\left(2 \cdot\left|C_{h}\right|\right)$ derived in (12), showing that the worst-case low-order harmonics obtained from EMTDC and measurement are bounded from above as expected. Moreover, comparing Fig. 11 with Fig. 9 highlights the importance of assessing PQ across a range of operating points as the operating point for which the largest harmonic magnitude occurs differs for each harmonic order.

\section{A Comment on the Aggregation of Current Harmonics in Wind Farms Consisting of Type-IV Wind Turbines}

The narrow range of the phase of the characteristic loworder odd voltage harmonics generated by the converter, exhibited in Fig. 10, has an important bearing on WPPs consisting of 


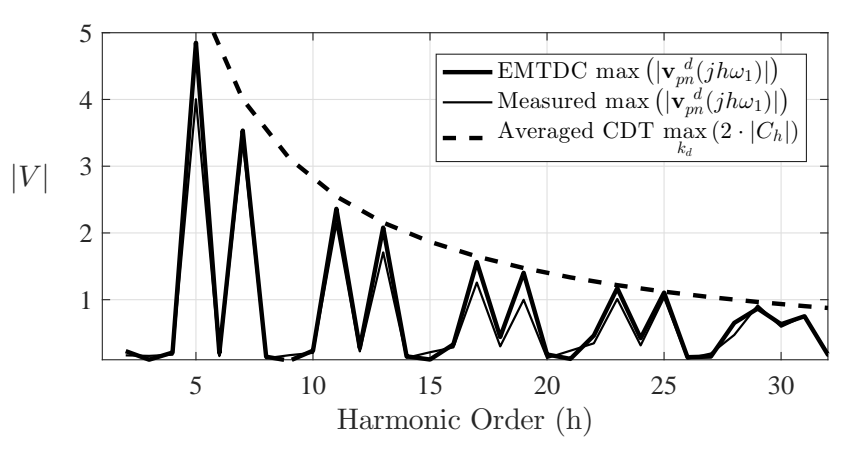

Fig. 11: Virtual spectrum of the maximal elements of $\left|\mathbf{v}_{p n}^{d}\left(j h \omega_{1}\right)\right|$ taken across all active power injections.

Type-IV wind turbines. Each wind turbine synchronizes to the grid voltage and injects a fundamental component of current to meet the active power requirement. The small voltage drop between each wind turbine in the collector system results in an approximately in-phase fundamental current injection by each wind turbine. Based on the findings in Section III-C, the converter generated odd voltage harmonics (at least for the assessed wind turbine) will tend to be in-phase, even across different active power injections, and thus inject harmonic currents that aggregate constructively at the point of common coupling. Accordingly, one would not expect the Rayleigh type attenuation of magnitude in the aggregated current, as discussed in [7], for these particular harmonics. Other wind turbines with different control or modulation strategies may exhibit different phase characteristics for low-order odd harmonics. These characteristics can likewise be extracted using the SD harmonic model.

\section{CONCLUSion}

A SD harmonic model of grid-connected VSCs has been introduced for decoupling converter generated voltage harmonics from voltage harmonics in the external grid. The SD harmonic model provides a general solution for assessing and computing PQ in individual and systems of Type-IV wind turbines respectively. The development of the SD harmonic model included a derivation of a baseband frequency response for regular-sampled PWM.

The SD harmonic model was used to assess the PQ of a $3 M W$ Type-IV wind turbine. The assessment showed that the magnitude of low-order converter generated odd voltage harmonics were dependent on the converter operating point. This aligned with an analysis of error pulses due to compensated dead-time obtained through an averaged value model. The phase of the aforementioned voltage harmonics were shown to be dependent on the phase of the fundamental component of current. This should be considered when computing PQ for WPPs consisting of Type-IV wind turbines.

\section{APPENDIX - BASEBAND FREQUENCY RESPONSE OF Regular-SAMPled Pulse Width Modulation}

Consider a normalized three-phase modulating signal, $\mathbf{v}_{a b c}^{m}(t)$, with a fundamental component of positive phase sequence and harmonic components of positive and negative phase sequence,

$$
\begin{aligned}
& \mathbf{v}_{a b c}^{m}(t)=\mathbf{v}_{a b c, 1}(t)+\mathbf{v}_{a b c, p}(t)+\mathbf{v}_{a b c, n}(t) \\
& =M_{1}\left[\begin{array}{c}
\cos \left(\omega_{1} t+\phi_{1}\right) \\
\cos \left(\omega_{1} t-2 \pi / 3+\phi_{1}\right) \\
\cos \left(\omega_{1} t+2 \pi / 3+\phi_{1}\right)
\end{array}\right]+\sum_{k=2}^{N} M_{p, k}\left[\begin{array}{c}
\cos \left(k \omega_{1} t+\phi_{p, k}\right) \\
\cos \left(k \omega_{1} t-2 \pi / 3+\phi_{p, k}\right) \\
\cos \left(k \omega_{1} t+2 \pi / 3+\phi_{p, k}\right)
\end{array}\right] \\
& +\sum_{k=2}^{N} M_{n, k}\left[\begin{array}{c}
\cos \left(k \omega_{1} t+\phi_{n, k}\right) \\
\cos \left(k \omega_{1} t+2 \pi / 3+\phi_{n, k}\right) \\
\cos \left(k \omega_{1} t-2 \pi / 3+\phi_{n, k}\right)
\end{array}\right]
\end{aligned}
$$

where $\left\{M_{1}, \phi_{1}\right\},\left\{M_{p, k}, \phi_{p, k}\right\}$ and $\left\{M_{n, k}, \phi_{n, k}\right\}$ are the magnitude and phase of the positive sequence fundamental, positive sequence harmonic and negative sequence harmonic components respectively, $k$ is the harmonic order. The modulating signal is applied to a two-level VSC using a regularsampled PWM scheme and connected to a balanced threephase load. The Poisson re-summation method proposed in [37] is applied to (13) giving the SD load voltage spectra,

$$
\begin{aligned}
& \mathbf{v}_{p n}^{l}(\omega)=\sum_{m, n} \frac{a_{m, n}}{3}\left[\begin{array}{l}
1+2 \cos \left(\frac{2 \pi}{3}\left(x_{1}-1+\sum_{k=2}^{N}\left(x_{p, k}-x_{n, k}\right)\right)\right) \\
1+2 \cos \left(\frac{2 \pi}{3}\left(x_{1}+1+\sum_{k=2}^{N}\left(x_{p, k}-x_{n, k}\right)\right)\right)
\end{array}\right] \\
& \cdot 2 \pi \delta\left(\omega-\Omega_{m n}\right) .
\end{aligned}
$$

where $a_{m, n}$ are the complex Fourier coefficients,

$$
\begin{aligned}
& a_{m, n}=\frac{4}{j \Omega_{m n} T_{c}} J_{x_{1}}\left(\frac{\Omega_{m n} M_{1} T_{c}}{4}\right) e^{j\left(x_{1} \phi_{1}+m \phi_{c}\right)} . \\
& \prod_{k=2}^{N} J_{x_{p, k}}\left(\frac{\Omega_{m n} M_{p, k} T_{c}}{4}\right) e^{j x_{p, k} \phi_{p, k}} J_{x_{n, k}}\left(\frac{\Omega_{m n} M_{n, k} T_{c}}{4}\right) e^{j x_{n, k} \phi_{n, k} .} \\
& j^{(m+n))} \cos \left(n \omega_{1} \frac{T_{c}}{4}(1-R)\right) e^{-j n \omega_{1} \frac{T_{c}}{4}(2-R)} .
\end{aligned}
$$

$T_{c}$ is the switching period, $\delta\left(\omega-\Omega_{m n}\right)$ is the delta function centered at $\omega=\Omega_{m n}, \Omega_{m n}=n \omega_{1}+m \omega_{c}$ is the frequency component for the $m$ th and $n$th indexes, $-\pi \leq \phi_{c} \leq \pi$ is the phase of the PWM carrier and $R$ is a binary parameter with $R=0$ for symmetrically sampled PWM and $R=1$ for asymmetrically sampled PWM. Additionally, $n=x_{1}+$ $\sum_{k=2}^{N}\left(k x_{p, k}+k x_{n, k}\right)$ where $x_{1}, x_{p, k}$, and $x_{n, k}$ are the indexes of the fundamental, $k$-th order positive sequence harmonic and $k$-th order negative sequence harmonic components in the modulating signal respectively. The baseband response for the $h$-th order positive sequence harmonic occurs for the indexes $m=0, x_{1}=0, x_{p, h}=1, x_{p, k}=0 \forall k \neq h, x_{n, k}=0 \forall k$ such that $\Omega_{m n}=h w_{1}$ and,

$$
\begin{aligned}
& \mathbf{v}_{p n}^{l}(\omega)=\frac{4}{h \omega_{1} T_{c}} J_{0}\left(h \omega_{1} \frac{M_{1} T_{c}}{4}\right) J_{1}\left(h \omega_{1} \frac{M_{p, h} T_{c}}{4}\right) e^{j \phi_{p, h}} . \\
& \prod_{\substack{k=2 \\
k \neq h}}^{N} J_{0}\left(h \omega_{1} \frac{M_{p, k} T_{c}}{4}\right) \prod_{k=2}^{N} J_{0}\left(h \omega_{1} \frac{M_{n, k} T_{c}}{4}\right) \cdot \\
& \cos \left(h \omega_{1} \frac{T_{c}}{4}(1-R)\right) e^{-j h \omega_{1} \frac{T_{c}}{4}(2-R)}\left[\begin{array}{l}
1 \\
0
\end{array}\right] \cdot 2 \pi \delta\left(\omega-h \omega_{1}\right) .
\end{aligned}
$$

Consider (16) for asymmetrically regular-sampled PWM where $R=1$ and $\omega_{s}=2 \omega_{c}$. The simplification $J_{0}(z) \approx 1$ is within $1 \%$ error if $z \leq 0.2$. If $z=h \omega_{1} M_{x, h} T_{c} / 4$, where $x=p, n$, then $M_{x, h} \leq 0.1273 \cdot q / h$ where $q=\omega_{c} / \omega_{1}$ is the pulse ratio. If the modulating signal is band-limited to 
$q \omega_{1}$ then $M_{x, q} \leq 0.1273$ is required for the simplification to hold. Similarly, the Bessel function $J_{1}(z) \approx z / 2$ when $z \ll 1$. If $z=h \omega_{1} M_{p, h} T_{c} / 4$ and $h=q$ then $J_{1}(z) \approx z / 2$ is within $1 \%$ error if $M_{p, h} \leq 0.63$. The loads supplied by power converters typically have low-pass impedance and converter control systems are normally designed to resonate at the fundamental frequency and dampen at harmonic frequencies. Therefore, under normal operation $M_{x, h} \ll 0.1$, and (16) simplifies to,

$$
\begin{gathered}
\mathbf{v}_{p n}^{l}(\omega)=J_{0}\left(h w_{1} \frac{M_{1} T_{c}}{4}\right) \cos \left(h \omega_{1} \frac{T_{c}}{4}(1-R)\right) e^{-j h \omega_{1} \frac{T_{c}}{4}(2-R) .} \\
\frac{M_{p, h}}{2} e^{j \phi_{p, h}}\left[\begin{array}{l}
1 \\
0
\end{array}\right] \cdot 2 \pi \delta\left(\omega-h w_{1}\right) .
\end{gathered}
$$

Similarly, it can be shown that the main baseband response for the $h$-th order negative sequence harmonic component in the modulating signal is given by,

$$
\begin{gathered}
\mathbf{v}_{p n}^{l}(\omega)=J_{0}\left(h w_{1} \frac{M_{1} T_{c}}{4}\right) \cos \left(h \omega_{1} \frac{T_{c}}{4}(1-R)\right) e^{-j h \omega_{1} \frac{T_{c}}{4}(2-R)} \\
\cdot \frac{M_{n, h}}{2} e^{j \phi_{n, h}}\left[\begin{array}{l}
0 \\
1
\end{array}\right] \cdot 2 \pi \delta\left(\omega-h w_{1}\right) .
\end{gathered}
$$

Equations (17) and (18) can be considered as the output harmonic phasors to the input harmonic phasors $M_{p, h} e^{j \phi_{p, h}}$ and $M_{n, h} e^{j \phi_{n, h}}$ respectively. The transfer matrix representation for the SD baseband frequency response is then,

$$
\mathbf{K}_{p n}=\left[\begin{array}{cc}
K_{M}(j \omega) & 0 \\
0 & K_{M}(j \omega)
\end{array}\right],
$$

where,

$$
K_{M}(j \omega)=J_{0}\left(\omega \frac{M_{1} T_{c}}{4}\right) \cos \left(\omega \frac{T_{c}}{4}(1-R)\right) e^{-j \omega \frac{T_{c}}{4}(2-R)} .
$$

\section{ACKNOWLEDGMENT}

This work was supported in part by Siemens Gamesa Renewable Energy (SGRE) and in part by the Innovations Fund, Denmark. The authors thank P. Brogan, L. Shuai and R. Sharma of SGRE and Professors V. Agelidis and J. Holbøll of DTU for their valuable input and discussions.

\section{REFERENCES}

[1] J. Arrillaga and N. R. Watson, "Power System Harmonics," John Wiley \& Sons Inc., Hoboken, NJ, USA, 2003.

[2] "Wind Turbines - Part 21: Measurement and assessment of power quality characteristics of grid connected wind turbines," International Electrotechnical Commission Standard IEC-61400-21, 2008.

[3] L. Sainz et al., "Deterministic and Stochastic Study of Wind Farm Harmonic Currents," IEEE Transactions on Energy Conversion, vol. 25, no. 4, pp. 1071-1080, Dec. 2010.

[4] S. T. Tentzerakis and S. A. Papathanassiou, "An Investigation of the Harmonic Emissions of Wind Turbines," IEEE Transactions on Energy Conversion, vol. 22, no. 1, pp. 150-158, Mar. 2007.

[5] K. Yang et al., "Decompositions of harmonic propagation in wind power plant," Electric Power Systems Research, vol. 141, pp. 84-90, Dec. 2016.

[6] K. Yang, M. H. J. Bollen, E. O. A. Larsson, and M. Wahlberg, "Measurements of harmonic emission versus active power from wind turbines," Electric Power Systems Research, vol. 108, pp. 304-314, Mar. 2014.

[7] S. A. Papathanassiou and M. P. Papadopoulos, "Harmonic Analysis in a Power System with Wind Generation," IEEE Transactions on Power Delivery, vol. 21, no. 4, pp. 2006-2016, Oct. 2006.

[8] K. Yang, M. H. J. Bollen, and E. O. A. Larsson, "Aggregation and Amplification of Wind-Turbine Harmonic Emission in a Wind Park," IEEE Transactions on Power Delivery, vol. 30, no. 2, pp. 791-799, Apr. 2015.
[9] F. Ghassemi and K.-L. Koo, "Equivalent Network for Wind Farm Harmonic Assessments," IEEE Transactions on Power Delivery, vol. 25, no. 3, pp. 1808-1815, Jul. 2010.

[10] Ł. H. Kocewiak et al., "Wind Turbine Harmonic Model and Its Application - Overview, Status and Outline of the New IEC Technical Report," in the 14th International Workshop on Large-Scale Integration of Wind Power into Power Systems, Brussels, 2015.

[11] L. Shuai et al., "Application of Type 4 Wind Turbine Harmonic Model for Wind Power Plant Harmonic Study," in the 15th International Workshop on Large-Scale Integration of Wind Power into Power Systems, Vienna, 2016.

[12] P. Brogan and N. Goldenbaum, "Harmonic Model of the Network Bridge Power Converter for Wind Turbine Harmonic Studies," in the 11th International Workshop on Large-Scale Integration of Wind Power into Power Systems, Lisbon, 2012.

[13] X. Wang, F. Blaabjerg, and W. Wu, "Modeling and Analysis of Harmonic Stability in an AC Power-Electronics-Based Power System," IEEE Transactions on Power Electronics, vol. 29, no. 12, pp. 6421-6432, Dec. 2014.

[14] J. L. Agorreta et al., "Modeling and Control of $N$-Paralleled GridConnected Inverters With LCL Filter Coupled Due to Grid Impedance in PV Plants," IEEE Transactions on Power Electronics, vol. 26, no. 3, pp. 770-785, Mar. 2011.

[15] J. Sun, "Impedance-Based Stability Criterion for Grid-Connected Inverters," IEEE Transactions on Power Electronics, vol. 26, no. 11, pp. 3075-3078, Nov. 2011.

[16] S. Zhang, S. Jiang, X. Lu, B. Ge, and F. Z. Peng, "Resonance Issues and Damping Techniques for Grid-Connected Inverters With Long Transmission Cable," IEEE Transactions on Power Electronics, vol. 29, no. 1, pp. 110-120, Jan. 2014.

[17] Z. Shuai, D. Liu, J. Shen, C. Tu, Y. Cheng, and A. Luo, "Series and Parallel Resonance Problem of Wideband Frequency Harmonic and Its Elimination Strategy," IEEE Transactions on Power Electronics, vol. 29, no. 4, pp. 1941-1952, Apr. 2014.

[18] L. Sainz, L. Monjo, J. Pedra, M. Cheah-Mane, J. Liang, and O. GomisBellmunt, "Effect of wind turbine converter control on wind power plant harmonic response and resonances," IET Electric Power Applications, vol. 11, no. 2, pp. 157-168, Feb. 2017.

[19] L. H. Kocewiak, J. Hjerrild, and C. L. Bak, "Wind turbine converter control interaction with complex wind farm systems," IET Renewable Power Generation, vol. 7, no. 4, pp. 380-389, Jul. 2013.

[20] L. Harnefors, M. Bongiorno, and S. Lundberg, "Input-Admittance Calculation and Shaping for Controlled Voltage-Source Converters," IEEE Transactions on Industrial Electronics, vol. 54, no. 6, pp. 3323-3334, Dec. 2007.

[21] X. Wang, Y. Li, F. Blaabjerg, and P. C. Loh, "Virtual-ImpedanceBased Control for Voltage-Source and Current-Source Converters," IEEE Transactions on Power Electronics, pp. 1-1, 2014.

[22] F. Wang, J. L. Duarte, M. A. M. Hendrix, and P. F. Ribeiro, "Modeling and Analysis of Grid Harmonic Distortion Impact of Aggregated DG Inverters," IEEE Transactions on Power Electronics, vol. 26, no. 3, pp. 786-797, Mar. 2011.

[23] J. H. R. Enslin and P. J. M. Heskes, "Harmonic Interaction Between a Large Number of Distributed Power Inverters and the Distribution Network," IEEE Transactions on Power Electronics, vol. 19, no. 6, pp. 1586-1593, Nov. 2004.

[24] L. Harnefors, "Modeling of Three-Phase Dynamic Systems Using Complex Transfer Functions and Transfer Matrices," IEEE Transactions on Industrial Electronics, vol. 54, no. 4, pp. 2239-2248, Aug. 2007.

[25] J. Sun, "Small-Signal Methods for AC Distributed Power Systems - A Review," IEEE Transactions on Power Electronics, vol. 24, no. 11, pp. 2545-2554, Nov. 2009.

[26] B. Wen, D. Boroyevich, R. Burgos, P. Mattavelli, and Z. Shen, "Analysis of D-Q Small-Signal Impedance of Grid-Tied Inverters," IEEE Transactions on Power Electronics, vol. 31, no. 1, pp. 675-687, Jan. 2016.

[27] M. Cespedes and Jian Sun, "Impedance Modeling and Analysis of Grid-Connected Voltage-Source Converters," IEEE Transactions on Power Electronics, vol. 29, no. 3, pp. 1254-1261, Mar. 2014.

[28] J. Yong, L. Chen, A. B. Nassif, and W. Xu, "A Frequency-Domain Harmonic Model for Compact Fluorescent Lamps,” IEEE Transactions on Power Delivery, vol. 25, no. 2, pp. 1182-1189, Apr. 2010.

[29] E. Mollerstedt and B. Bernhardsson, "Out of control because of harmonics-an analysis of the harmonic response of an inverter locomotive," Control Systems, IEEE, vol. 20, no. 4, pp. 70-81, 2000.

[30] J. J. Rico, M. Madrigal, and E. Acha, "Dynamic harmonic evolution using the extended harmonic domain," IEEE Transactions on Power Delivery, vol. 18, no. 2, pp. 587-594, Apr. 2003. 
[31] M. Esparza, J. Segundo-Ramirez, J. B. Kwon, X. Wang, and F. Blaabjerg, "Modeling of VSC-Based Power Systems in The Extended Harmonic Domain," IEEE Transactions on Power Electronics, pp. 1-1, 2017.

[32] D. Leggate and R.J. Kerkman, "Pulse-Based Dead-Time Compensator for PWM Voltage Inverters," IEEE Transactions on Industrial Electronics, vol. 44, no. 2, pp. 191-197, 1997.

[33] S.-G. Jeong and M.-H. Park, "The analysis and compensation of dead-time effects in PWM inverters," IEEE Transactions on Industrial Electronics, vol. 38, no. 2, pp. 108-114, 1991.

[34] G. Grandi, J. Loncarski, and R. Seebacher, "Effects of current ripple on dead-time distortion in three-phase voltage source inverters," in Energy Conference and Exhibition (ENERGYCON), 2012 IEEE International, 2012, pp. 207-212.

[35] C. D. Townsend, G. Mirzaeva, and G. C. Goodwin, "Deadtime Compensation for Model Predictive Control of Power Inverters," IEEE Transactions on Power Electronics, vol. 32, no. 9, pp. 7325-7337, Sep. 2017.

[36] D. G. Holmes and T. A. Lipo, "Pulse Width Modulation for Power Converters: Principles and Practice," Wiley-IEEE Press, Hoboken, NJ, USA, 2003.

[37] S. M. Cox, "Voltage and current spectra for a single-phase voltage source inverter," IMA Journal of Applied Mathematics, vol. 74, no. 5, pp. 782-805, Oct. 2009.

[38] Z. Song and D. V. Sarwate, "The frequency spectrum of pulse width modulated signals," Signal Processing, vol. 83, no. 10, pp. 2227-2258, Oct. 2003.

[39] "IEEE Recommended Practices and Requirements for Harmonic Control in Electrical Power Systems," IEEE Standard 519, 2014.

[40] M. Cespedes and J. Sun, "Three-phase impedance measurement for system stability analysis," in Control and Modeling for Power Electronics (COMPEL), 2013 IEEE 14th Workshop on, 2013, pp. 1-6.

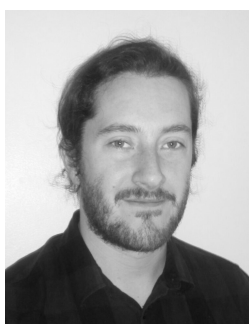

Emerson Guest received the Bachelor degree (2010) from the University of Newcastle, Newcastle, Australia, and the M.Sc. degree (2014) from the Technical University of Denmark, Kgs. Lyngby, Denmark, where he is currently working towards the Industrial $\mathrm{Ph} . \mathrm{D}$. degree, all in electrical engineering.

His research interests include harmonic modeling of grid-connected converters, active filtering and real-time optimization of power electronic systems.

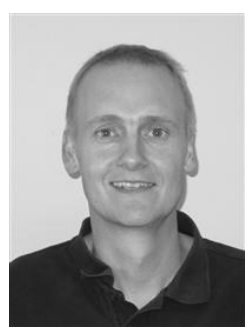

Kim H. Jensen holds M.Sc. (1999) and Ph.D. (2003) degrees from the Technical University of Denmark, Kgs. Lyngby, Denmark. He has been working in a transmission company (NESA), consultant company (Elsam Engineering) and now Siemens Gamesa Renewable Energy. During his career he has worked with transmission system planning, network designs, wind turbine modeling, grid code studies, harmonic analysis and stability studies. He has also worked as a technical specialist on a number of wind farm integration projects.

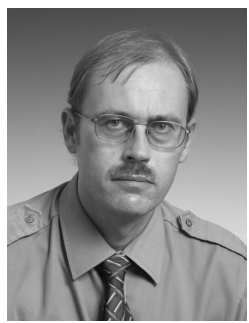

Tonny W. Rasmussen holds M.Sc. (1993) and Industrial Ph.D. (1998) degrees from the Technical University of Denmark, Kgs. Lyngby, Denmark. $\mathrm{He}$ is an Associate Professor for the Centre for Electric Power and Energy, Technical University of Denmark, Kgs. Lyngby, and a Member of the IEEE Power Electronics Society.

His research interests include power electronic converter topologies, converter control and gridconnected applications of converters. 